\title{
SAÚDE MENTAL NA ESCOLA: UM OLHAR SOBRE O TRANSTORNO DO ESPECTRO DO AUTISMO
}

\section{MENTAL HEALTH AT SCHOOL: A LOOK AT AUTISM SPECTRUM DISORDER}

\author{
José Marciel Araújo Porcino \\ Bacharelado em Psicologia pelo Centro \\ Universitário de Patos-UNIFIP (2015), \\ Especialista em Saúde Mental, e Licenciando \\ o 6 Período do curso de Pedagogia da \\ Universidade Federal da Paraíba-UFPB na \\ modalidade de Educação a Distância-EAD. \\ E-mail: leicram ap@hotmail.com
}

Jadcely Maria Viturino Serafim Porcino

Graduada em Fisioterapia pela Universidade Federal da Paraíba-UFPB, Especialista em

Saúde Mental pelo Centro Universitário de

Patos-UNIFIP

E-mail: jadcelymvs@gmail.com

\section{Valéria Amanda Jerônimo Pereira}

Graduada em Psicologia Pela Universidade

Federal da Paraíba-UFPB, pós graduanda em Neuropsicologia Pelo Centro Universitário de João Pessoa- UNIPÊ.

E-mail: valeriaamanda@hotmail.com

Silmaria Bezerra Porcino

Mestre em Ciências da Educação Pelo

Instituto Superior de Educação Professora

Lúcia Dantas-ISEL e Graduada em Serviço Social Pela Universidade Federal da ParaíbaUFPB, Brasil. E-mail: siwmarya@hotmail.com

\section{Resumo}

Esse estudo apresenta-se como uma análise compreensiva, descritiva, crítica e reflexiva sobre a saúde mental na escola com olhar voltada à criança no Transtorno do Espectro do Autismo (TEA). No presente estudo busca-se descrever a saúde mental no âmbito escolar, os fazeres e saberes relacionados às práxis de professores frente ao aluno no TEA. Adota-se como abordagem, a metodologia de revisão de literatura, onde se utilizou livros, teses, dissertações e artigos indexados Scielo e Gooogle acadêmico no período de novembro de 2018 a maio de 2019. Ainda foram utilizadas as palavras-chave: Transtorno do Espectro do Autismo na escola, metodologia de ensino em saúde mental de pessoa no TEA no ambiente escolar, saúde mental de criança no TEA na escola. Os achados apontam que são poucos os estudos que 
evidenciam a importância da saúde mental no TEA. Alguns destacam os problemas de ordem de biológica, hereditária, genética ou quadro psicossociais conflitantes, e outras se voltam para diagnóstico e intervenção. Contudo, observa-se que pesquisas apontam a relevância dos fazeres e saberes em saúde mental no âmbito escolar, onde o professor/mediador pode desenvolver suas práxis baseadas em evidências de teoria do desenvolvimento humano, cognitiva-comportamental, psicanalítica e psicodinâmica. Ações essas desenvolvidas e conduzidas por práxis de ateliês, salas ambientes, música e teatro. Conclui-se, que essas ações terapêuticas voltadas para interação, dialética e a percepção acerca do aluno no TEA. Ainda, trabalham conteúdo cognitivocomportamental, afetivo, emocional e social, de modo que é considerado genuinamente um fator em saúde mental, pois, todos interagem entre si.

Palavras-chave: saúde mental; fazeres e saberes; ações terapêuticas.

\section{Abstract}

This study presents itself as a comprehensive, descriptive, critical and reflective analysis of mental health in school with a child-focused look on Autism Spectrum Disorder (ASD). This study aims to describe the mental health in the school environment, the doings and knowledge related to the teachers' praxis before the student in the TEA. The approach adopted is the literature review methodology, which used books, theses, dissertations and indexed articles Scielo and Gooogle academic from November 2018 to May 2019. The following keywords were also used: Spectrum Disorder of Autism at school, methodology of teaching mental health of person in ASD in the school environment, child mental health in ASD in school. The findings indicate that there are few studies that show the importance of mental health in ASD. Some highlight conflicting biological, hereditary, genetic, or psychosocial issues, and others focus on diagnosis and intervention. However, it is observed that research points to the relevance of mental health practices and knowledge in the school environment, where the teacher / mediator can develop their praxis based on evidence of human development theory, cognitive behavior, psychoanalytic and psychodynamic. These actions are developed and conducted by praxis of studios, ambient rooms, music and theater. It is concluded that these therapeutic actions aimed at interaction, dialectics and the perception about the student in ASD. Still, they work on cognitive-behavioral, affective, emotional and social content, so it is considered genuinely a factor in mental health, because all interact with each other

Keywords: mental health; actions and knowledge; therapeutic actions.

\section{Introdução}

A saúde mental é considerada um processo de construção em face ao fenômeno da personalidade, que por sua natureza intrínseca estar ancorado em fatores: biológico, hereditário, cognitivo, afetivo, emocional, psíquico, social, histórico, econômico e fenomenológico presente na vida de uma pessoa (DALGALARRONDO, 2018), sendo a saúde mental um elemento imprescindível, de forma que se configura em um elo que conectam todos esses fatores em um só.

Circunscrevendo, nesse contexto, o Transtorno do Espectro do Autismo (TEA) é uma síndrome caracterizada por alteração no neurodesenvolvimento, de modo a 
provocar dificuldades em se relacionar, problemas na interação, dificuldade na linguagem ou na fala, nas expressões gestuais e corporais, ou comportamentos repetitivos e estereotipados entre outros fatores (TAMANAHA, PERISSINOTO, CHIARI, 2008; MEIMES, SALDANHA \& BOSA, 2015), são os principais diagnósticos encontrados pela criança com Transtorno do Espectro do Autismo (TEA).

Nesse sentido, observa-se que a saúde mental da criança no TEA, requisita de cuidados continuados da família, dos profissionais da área de saúde, da sociedade e área da educação. Este último, por sua grandeza no meio escolar, pode-se tornar o ponto essencial da conduta facilitadora no manejo de saúde mental. (DO NASCIMENTO BARRO, SALES, PIOVESAN; 2018).

A escola por ser um ambiente favorável para o desenvolvimento das habilidades de aprendizagens do ser humano torna-se um ambiente vislumbrado para afloração da saúde mental. Assim, nota-se que nessa área requer um olhar crítico e reflexivo frente às novas configurações contemporâneas e suas implicações no campo da saúde mental. (DO NASCIMENTO BARRO, SALES, PIOVESAN; 2018).

Deste modo, percebe-se a necessidade e a pertinência em descrever a saúde mental inserida no ambiente escolar, de modo a descrever as práxis dos profissionais da área da educação frente à saúde mental do portador do TEA no âmbito escolar, corroborando para efetivação de atividades e a importância da saúde mental. (RIBAS, TERRA, ERDMANN;2005).

Igualmente, compreende-se que quando não é direcionada uma reflexão crítica sobre a saúde mental no ambiente escolar, percebe-se a inexistência do foco no trabalho na promoção de saúde mental frente às possíveis problemáticas e suas demandas, onde se observa que as mesmas podem acarretar em desequilíbrio psíquico, e, consequentemente trazer danos à saúde mental e integral. (VIEIRA et al, 2014).

Nesse contexto, nota-se que na contemporaneidade, a saúde mental na escola é um dos vieses norteadores para o desenvolvimento humano, pois sua denotação cientifica no campo educacional pode implicar nas mais variadas formas e conteúdo da saúde psíquica do ser humano. Os quais podem ser positivos ou negativos. Assim, dessa forma, Ramires et al (2009) destaca que fica evidente, também, a necessidade de estudos que contemplem o tema da saúde mental de crianças e que ofereçam um panorama da produção científica existente.

De tal modo, que é na escola que se configura um ambiente favorável para desenvolvimento da saúde mental. E é neste cenário que pode emergir e ser trabalhado as aflorações das múltiplas habilidades do bem-estar da pessoa com 
sofrimento psicológico, distúrbios psiquiátricos ou transtornos do neurodesenvolvimento. (VIEIRA et al, 2014).

Diante disso, surgem os seguintes questionamentos: como é descrita a saúde mental na escola de pessoas com Transtorno do Espectro do Autismo e como é trabalhada a saúde mental desse público na escola? Nesse sentido, o presente trabalho busca descrever a saúde mental de pessoas com TEA no âmbito escolar.

No que tange à metodologia esta é uma pesquisa qualitativa de cunho bibliográfico e exploratória constituindo-se em uma revisão crítica e reflexiva da literatura sobre a descrição da saúde mental de criança com o Transtorno do Espectro do Autismo inserido no ambiente escolar. Os dados utilizados nesse trabalho foram oriundos de pesquisa realizada em rede nas bases de dados indexadas Scielo e Google acadêmico, livros, teses, dissertações e artigos de relevo para construção de uma base teórica sólida, realizada no período de novembro de 2018 a maio de 2019. Ainda foram utilizadas as palavras Chaves: TEA na escola, metodologia de ensino de pessoa com TEA no ambiente escolar, descrição de ensino a pessoa com TEA, saúde mental de criança/pessoa com TEA na escola.

No que se refere à concepção da pesquisa de revisão de literatura, Gil (2002) aponta que esse método de pesquisa é uma base bibliográfica que tem como objetivo corroborar com um problema de pesquisa, de maneira a propor a partir de material já elucidado, solução ao objeto problemático. Assim, nessa visão, o presente estudo será respondido através dessa abordagem, de modo a propor uma reflexão crítica e construtivista diante dessa demanda cada vez mais presente nos dias atuais.

\section{Educação especial e o transtorno do espectro do autismo}

O advento das Políticas Públicas de Inclusão Educacional Escolar, trouxe ao Brasil a possibilidades de potencializar o desenvolvimento de aprendizagens da pessoa com algum tipo de deficiência (BRASIL, 2007), onde suas ações visam contemplar o planejamento pedagógico e a execução de atividades diante desse "ser". Garantindo assim, todos os direitos conquistados ao longo dos anos, tais como reivindicados pela: Declaração Universal dos Direitos Humanos (1948), a Constituição Federal de (1988), a Declaração de Jomtien (1990) e a Declaração de Salamanca (1994) que corroboraram para institucionalização das Políticas Nacionais de Educação 
Especial na Perspectiva da Educação Inclusiva (2008). (DE PAULA NUNES, DE AZEVEDO \& SCHMIDT, 2013).

Diante do exposto, Do Nascimento Barro, Sales, Piovesan $(2018$, p1.) enfatiza que "A Educação atualmente possui sistemas de ensino que devem fornecer e organizar condições de acesso que favoreçam a aprendizagem, atendendo as necessidades educacionais de todos os alunos, respondendo a uma política de inclusão".(DO NASCIMENTO BARRO, SALES, PIOVESAN, 2018 p.1).

Perfazendo desse caminho, Maior (2018, pp 6-7) destaca que foi a partir:

A partir da Lei 12764/2012, as pessoas com transtorno do espectro autista (TEA) passaram a ser consideradas pessoas com deficiência. Elas apresentam deficiência significativa na comunicação e na interação social (BRASIL, 2012). Os casos podem variar desde não aprender a falar e ter deficiência intelectual profunda até não ter deficiência intelectual e conviver na comunidade, seguindo suas próprias rotinas. Também se caracterizam por comportamento repetitivo (balançar o corpo, as mãos, gritar) e áreas restritas de interesse. Tal como em outros casos de deficiência, são pessoas em risco de violência e necessitam de 7 atendimento especializado dos órgãos de defesa de direitos e de segurança pública.

O que se nota é que essas leis trouxeram avanços significativos ao propor adequações à pessoa no quadro do TEA, além de assegurar o processo de inclusão, viabilizando a entrada de um profissional especializado para mediar e facilitar 0 processo de inclusão-ensino-aprendizagem ao aluno. (GIARDINETTO, 2009; PARRA, 2009; DE PAULA NUNES, DE AZEVEDO \& SCHMIDT, 2013).

\section{Pressupostos históricos acerca do autismo}

Tudo percorre a análise dos registros fundamentados por pesquisadores que antes submeteram a escrever e registar seus dados para que outros estudiosos possam resgatar, analisar e descrever seus feitos teóricos e metodológicos. Em função disso, em consonância com a história do autismo, podemos citar que foi o eminente teórico Bleuler (1911) o primeiro a usar a expressão "autismo" ao enfatizar em estudo que a pessoa acometida por essa síndrome apresentava dificuldade em manter contato com a realidade e a comunicação. (GADIA, TUCHMAN, ROTTA, 2004).

Posteriormente, Kanner em 1943, descreveu em estudo realizado com 11 crianças que apresentavam em comum um comportamento atípico os quais não estabeleciam contato afetivo e interpessoal, sendo considerada uma psicopatologia incomum, daí, o mesmo uso a mesma expressão "autismo" para estabelecer um possível diagnóstico. (GADIA, TUCHMAN, ROTTA, 2004).

Em 1944, Asperger, descreveu em seus relatos características semelhantes ao autismo de Kanner, onde a eriança apresentava difieuldade de comunieação social, 
porém, mantinha o estado de interação normal. (GADIA, TUCHMAN, ROTTA, 2004), em outras palavras, Porcino et al (2018, p.248), corroboram com essa perspectiva a considerar que essa semelhança se correlacionam ao ponto de:

Sendo assim, nota-se que os estudos apontam existência de certa correlação entre Autismo e síndrome de Asperger, de forma que os sinais e sintomas são muitos semelhantes. Porém, na sintomatologia do Asperger o grau de alterações é reduzido levando em conta os níveis da aprendizagem. Enquanto que no autismo se eleva o grau de dificuldade de aprendizagem.

Diante dessas concepções, nota-se que os sinais e sintomas do autismo se apresentam através de déficit na comunicação, interação social e comportamento estereotipado. (KLIN, 2006; BRASIL, 2015). No entanto, Brasil (2015) elenca outros aspectos subjacentes ao autismo, tanto é que corrente cognitivistas apontam para existência de problemas na área da cognição.

\subsection{Descrições da saúde mental de pessoas com transtorno do espectro do autismo no âmbito escolar}

No que tange a saúde mental de criança com o quadro no TEA no ambiente escolar, percebe-se que são poucos os estudos que evidenciam ou trabalham com essa noção que é primordial para o funcionamento da pessoa como um todo. Assim, dessa forma, acredita-se que o desenvolvimento mental da criança se insere num processo contínuo de desenvolver as habilidades cognitivas e a interação entre o diálogo do pensamento, comportamento e das emoções (COELHO \& PISONI, 2012; FONSECA, 2016).

Corroborando com essa perceptiva, Fonseca (2016) chama atenção para o sofrimento emocional como uns dos problemas de saúde mental na escola. Dessa forma, que o professor deveria compactuar junto à gestão ações de práxis que possam favorecer condições para o desenvolvimento da aprendizagem emocional e afetiva.

Nesse sentido, observa-se que a saúde mental no âmbito escolar, existe na interação desses fatores. Deste modo, para que possa existir satisfatoriamente, a saúde psíquica é imprescindível articular saberes e fazeres na compreensão do funcionamento psíquico e suas implicações no dia a dia.

Por outro lado, Ramires, et al (2009, p.312) apontam em estudos que:

Os fatores de risco para os problemas de saúde mental na infância, incluem: os fatores biológicos (anormalidades biológicas do sistema nervoso central causadas por lesões, infecções, desnutrição ou exposição à toxinas), os genéticos (história familiar de depressão, por exemplo), os psicossociais (disfunções na vida familiar, discórdia conjugal grave, psicopatologia materna, criminalidade paterna, falta de laços afetivos entre pais e filhos), os eventos de vida estressantes (morte ou separação dos pais) e a exposição aos maus- 
tratos (negligência, abuso físico, psicológico ou sexual) (United States Department of Health and Human Services, 1999).

Diante do exposto, pode-se notar que o autismo ao pertencer aos aspectos do desenvolvimento, dos fatores biológicos e sociais e sua interface com a ambientação da escola, torna-se um cenário onde podem dificultar a saúde mental da criança. Pois, o autismo aparece em primeiro lugar em estudos no que se refere à saúde mental de crianças (RAMIRES \& et al, 2009), porém, na sua conjuntura essas pesquisas se aglomeram em situações clínicas e não abrangem uma interdisciplinaridade evolutiva e contínua.

Seguindo ainda os resultados destacados por Ramires et al (2009) as abordagens de intervenção frente à criança no espectro do autismo, circunscrevem em modelo teórico psicanalítico ao trabalho das funções do grupo inserido em instituição. Desta forma, elenca-se a escola como uma instituição voltada para desempenhar o desenvolvimento da pessoa.

Nesse sentido, compreende-se a saúde mental da criança no TEA, através do funcionamento psicodinâmico diante das funções do ego, da linguagem, e o desempenho social, cognitivo e os modos das habilidades de atenção compartilhada. O que se aproximam com o âmbito escolar. (RAMIRES et al , 2009)

Corroborando com essa perspectiva, Assis et al (2009) enfatizam que o autismo pertence ao quadro dos transtornos do desenvolvimento. Sendo considerado, portanto, um problema de saúde mental. Desta forma, os problemas psíquicos em sala de aula se caracterizam por dificuldade em interagir e estabelecer contatos afetivoemocionais, comportamentos atípicos ou agressivos que podem prejudicar no desenvolvimento escolar. (TAMANAHA, PERISSINOTO, CHIARI, 2008; MEIMES, SALDANHA \& BOSA, 2015).

\subsection{Fazeres e saberes em saúde mental relacionados às práxis de professores frente a pessoa com transtorno do espectro do autismo}

O estado integral do funcionamento psíquico de uma pessoa pode ser considerado um processo dinâmico, situacional e relacional conduzido pela cognição e a percepção que cada um tem sobre o mundo, as pessoas e de si mesmo. (BECK \& ALFORD, 2000). Assim, corroborando com essa perspectiva, Goldberg (2002) aponta que a percepção que os professores detêm sobre o autismo e suas implicações no cenário educacional, torna-se fundamental no processo de construção de práxis educacionais que promovam o desenvolvimento de suas habilidades. 
Por outro lado, nota-se que as práxis psicopedagógicas frente ao aluno com TEA em classes comuns, são executadas a grosso modo, pelo modelo cognitivocomportamental. Não que ofereçam subsídios em saúde mental e sua dialética com aprendizagem. A questão que se observa, é que a família e a pessoa com TEA são excluídas da escuta terapêutica integrais. Ou seja, não existe uma interação desde o diagnóstico e as práxis dos professores com os familiares, alunos e a gestão escolar, que são princípios fundamentais no processo de incluir na área escolar. (PRESTES 2009; BRAGA, 2009; DE PAULA NUNES, DE AZEVEDO \& SCHMIDT, 2013).

Neste contexto, observa-se que um dos fazeres que os professores devem aperfeiçoar em sala de aula para que a saúde mental da pessoa com TEA possa se desenvolver, é compreender as manifestações cognitivas, psíquicas e comportamentais ancoradas no modelo cognitivo de percepção no campo educacional (GOLDBERG, 2002), de modo a considerar todos os envolvidos nesse processo. Cabendo assim, ao professor/mediador atuar conforme as percepções que tem sobre o aluno com TEA e as relações estabelecidas com a família, onde o mesmo pensará, sentirá e agirá em função da realidade e do desenvolvimento de possibilidades e potencialidades dos seus alunos.

Compartilhando com essa visão, Sanches e Siqueira (2016), em pesquisa de estudo de caso, evidenciaram a importância do "trabalho com pessoas que apresentam autismo no âmbito da escola", pois, consideram fundamental que os professores ou mediadores, compreendam o real sentido de trabalhar as condições, as particularidades e as potencialidades da aprendizagem.

Para alcançar esses objetivos, Oliveira (2018) salienta que é imprescindível construir ou reconstruir objetivos e intervenções focadas na interação, mediação e a participação na dialética interacionista frente ao autismo, de modo que:

O objetivo das intervenções pedagógicas na abordagem construtivista e histórico-cultural, deve partir da possibilidade de desenvolvimento, associadas à interação da criança com o meio e com os conhecimentos a serem assimilados no contexto social escolar, dessa forma a criança com autismo deve ser motivada a participar das atividades cotidianas a sua maneira, sendo respeitada e compreendida.

Deste modo, promover ações terapêuticas no campo da saúde mental nos dias atuais é promover o desenvolvimento humano. Pois é nesse cenário da escola que a saúde mental deveria ser vista como elo norteador para desenvolvê-lo como pessoa, de modo que, esse ambiente é mais um que as relações e interações humanas entram em conflitos no processo dinâmico de descobertas.

Igualmente, percebe-se que nesse âmbito o funcionamento psíquico da pessoa adentra em manifestações psíquicas e comportamentais, cognitivas, afetivas, sociais e 
fenomenológicas, podendo estas ser positivas ou negativas. Assim, nesse sentido, nota-se que a educação no seio escolar é considerada uma estratégia terapêutica para a realização, manutenção e aperfeiçoamento das funções psíquicas. Deste modo, Kupfer (2001) enfatiza que a educação é função terapêutica, e suas ações devem olhar para as práxis interdisciplinares.

Na visão psicanalítica, os arranjos terapêuticos da escola, são em sua essência constituídos e mediados pelo professor, pois ao introduzir a técnica de transferência em sala de aula, o professor passa a mediar às manifestações psíquicas e comportamentais da criança com TEA. (DANTAS, 2009; DE PAULA NUNES, DE AZEVEDO \& SCHMIDT, 2013).

Diante desse contexto, ressalta-se a importância de considerar outros modelos teóricos na construção da mediação em saúde mental, onde se insere a pessoa com TEA, a qual muitas vezes, não é compreendida diante da sua dificuldade. Porém, é importante destacar que uma das práxis dos educadores na contemporaneidade frente à pessoa com TEA, são atividades que contemplem ações terapêuticas (MARTINS, DE ALMEIDA \& DE FREITAS, 2007).

Assim, dessa forma, Martins, Almeida e Freitas (2007) apontam que trabalhar atividades como: "ateliês ou salas-ambientes," diante de pessoas com TEA, é considerado um recurso terapêutico, pois essas práxis tende-se a corresponder às particularidades e singularidades de cada pessoa (MARTINS, DE ALMEIDA \& DE FREITAS, 2007), de modo que, nessas atividades, são utilizados múltiplos meios para impelir o desenvolvimento da linguagem.

Nesse sentido, Serra (2010) descreve que as implicações terapêuticas no âmbito escolar diante da pessoa com TEA, se darem no processo de ensinoaprendizagem ou na ressignificação da construção, ou ainda, na reconstrução da mediação da aprendizagem. Assim sendo, o manejo na educação e o cuidado em aplicar, desenvolver e facilitar os arranjos educacionais teóricos e práticos presentes nesse processo torna um método da própria saúde mental, uma vez que o autismo é compreendido através de correntes psicológicas ou psiquiátricas, de tal modo que a educação pode e deve ser usada como técnica.

Corroborando com essa perspectiva, Cavalcante (2018) enfatiza que "a educação é fundamental na formação do indivíduo no fator emocional, intelectual e social". Ou seja, a saúde psíquica se relaciona, percorre e interage mediante a esses fatores.

Indo de encontro com essa perspectiva, Bagarollo, Ribeiro e Panhoca (2013), ao destacarem a importância de todas as dimensões do ser humano, enfatizam que a 
dinâmica interacionista estabelecida no "brincar" torna-se um dos fazeres e saberes no cenário escolar. Pois, essa abordagem quando estabelecidas em sala de aula pode proporcionar atividades terapêuticas, de modo que implica no desenvolvimento de múltiplos aspectos relevante a criança, e, especificamente aquelas no TEA (ALVES \& ALVES, 2016), de modo geral, essas atividades terapêuticas podem trabalhar conteúdos: cognitivo, comportamental, afetivo, emocional e social. Os quais podem ser considerados genuinamente um fator em saúde mental, pois, todos interagem entre si.

\section{Considerações finais}

Diante dessa análise compreensiva, crítica e reflexiva da saúde mental na escola com um olhar voltado ao aluno com Transtorno do Espectro do Autismo (TEA). Constataram-se na sua descrição que os fazeres e os saberes no âmbito escolar frente a esse público, parte das políticas públicas, teorias do desenvolvimento humano, psicológicas, cognitivas, comportamentais, psicodinâmicas e psicanalistas as quais auxiliam no manejo psicopedagógico.

Ao reconhecer a importância das ações terapêuticas voltadas aos fazeres e saberes em saúde mental no âmbito escolar, onde o professor/mediador pode desenvolver suas práxis baseadas em evidência de práxis de ateliês, salas ambientes, música, teatro, cozinha e brincar. Acredita-se que essas atividades podem subsidiar ações terapêuticas voltadas para a interação, a dialética e a percepção acerca do aluno no TEA.

Assim, dessa forma, essas ações terapêuticas podem trabalhar conteúdos: cognitivo, comportamental, afetivo, emocional e social, de modo que são considerados genuinamente um fator em saúde mental, pois, todos interagem entre si. Descreve-se, portanto, que em todos os casos, recomenda-se que a gestão escolar e os profissionais possam compreender a relevância do funcionamento psíquico da criança com deficiência. Pois, percebe-se que trabalhar ações terapêuticas que contemplem a saúde mental na escola é essencial para o desenvolvimento humano integral.

Contudo, tão importante quanto à saúde mental e os fazeres e saberes a criança com deficiência no TEA é a importância da capacitação continuada do corpo docente, para que, haja uma visão diferenciada, humanizada, de maneira que assegure o acompanhamento dela no ambiente escola.

De maneira geral, pode dizer que essa descrição significa a sensibilização e o reconhecimento da saúde mental nesse contexto. É assim que os fazeres e saberes são colocados na práxis mediante a interação, o diálogo e aproximação das vivencias 
Espera-se que a partir desse estudo, outros pesquisadores possam corroborar numa perspectiva integrativa da saúda mental no TEA. Por certo, a mobilizar a sociedade num caminho em educação psíquica. Ainda, espera-se que essa pesquisa possa contribuir para consolidação da efetivação de práxis em saúde mental no ambiente escolar frente à pessoa com TEA.

Finalmente, ressalta-se que a saúde mental é um fenômeno vislumbrador presente na vida, de maneira que quando manejada satisfatoriamente pode ser construído e reconstruído formas e conteúdo que contribuam para desenvolver da saúde psíquica aos olhos de um, em um outro, que possa mediar e facilitar a compreensão integrativa no TEA.

Assim, dessa forma, lutamos por uma educação dialética em saúde mental na escola, onde a gestão escolar, os professores e principalmente a criança possam compreender a magnitude dos fenômenos psíquicos na vida. Pois, são através deles que identificamos, conhecemos, autoanalisamos, refletimos, construímos, reconstruímos, significamos e resignificamos a saúde mental e a nossa existência.

\section{Referências}

. Declaração de Salamanca e linha de ação sobre necessidades educativas especiais. Brasília: UNESCO, 1994.

. Ministério da Educação. Secretaria de Educação Especial. Política Nacional de Educação Especial na Perspectiva da Educação Inclusiva. Brasília, 2008a. Disponível em: <www.mec.gov.br>. Acesso em: 17 abril 2019.

- Ministério da Saúde. Secretaria de Atenção à Saúde. Departamento de Ações Programáticas Estratégicas. Diretrizes de Atenção à Reabilitação da Pessoa com Transtornos do Espectro do Autismo / Ministério da Saúde, Secretaria de Atenção à Saúde, Departamento de Ações Programáticas Estratégicas. Brasília: Ministério da Saúde, 2015. Disponível em: <http://portal.saude.gov.br/portal/arquivos/pdf/dir_tea.pdf. Acesso em: 17 de abril 2019.

ALVES, Maria Alina; ALVES, Marcelo Paraíso. O brincar como intervenção pedagógica nos transtornos do espectro do autismo. Revista Práxis, 2016, 8.1 (Sup).

BAGAROLLO, M. F.; RIBEIRO, Vanessa Veis; PANHOCA, Ivone. O brincar de uma criança autista sob a ótica da perspectiva hitórico-cultural. Revista brasileira de educação especial, v. 19, n. 1, p. 107-120, março, 2013.

BECK, A \& ALFORD, B. (2000) O poder integrador da terapia cognitiva (M.C. Monteiro, Trad.) Porto Alegre: Artes Médicas. (Original Publicado em 2000).

BRAGA, I. S. Teorizando as práticas de atendimento à pessoa com autismo na rede de escolas públicas do Distrito Federal. Dissertação (Mestrado em Psicologia). Programa de Pós-graduação em Psicologia da Universidade Católica de Brasília, Brasília - DF, 2009. 
BRASIL, Lei № 12.764 de 27 de dezembro de 2012. Disponível em: https://www.planalto.gov.br/ccivil 03/ ato2011-2014/2012/lei//12764.htm Acesso em: 16 abril 2019.

BRASIL. Declaração Mundial sobre Educação para Todos: plano de ação para satisfazer as necessidades básicas de aprendizagem. UNESCO, Jomtiem/Tailândia, 1990.

BRASIL. Ministério da Educação. Plano de Desenvolvimento da Educação: razões, princípios e programas. Brasília: MEC, 2007.

CAMARGO, Síglia Pimentel Höher; BOSA, Cleonice Alves. Competência social, inclusão escolar e autismo: revisão crítica da literatura. Psicologia \& sociedade. São Paulo SP. Vol. 21, n. 1 (jan./abr. 2009), p. 65-74., 2009.

Cavalcante, Jociana Matildes: A Inclusão no contexto escolar. In: Dickmann, Ivanio (Org.). Rumos da educação vol 5. 1a ed. São Paulo: Dialogar, 2018.

COELHO, L., \& PISONI, S. (2012). Vigotski: sua teoria e influência na educação.

Revista Modelos-FACOS/CNEC. Osório, n. 2, v. 1, p.144-152.

DALGALARRONDO, Paulo. Psicopatologia e semiologia dos transtornos mentais.

Artmed Editora, 2018.

DANTAS, A. P. Entre o educar e o constituir: efeitos possíveis da educação de uma criança com transtorno grave de desenvolvimento. Dissertação (Mestrado em

Psicologia Clínica). PUC - São Paulo, 2009.

DE PAULA NUNES, Debora Regina; DE AZEVEDO, Mariana Queiroz Orrico;

SCHMIDT, Carlo. Inclusão educacional de pessoas com Autismo no Brasil: uma revisão da literatura. Revista Educação Especial, 2013, 26.47: 557-572.

DO NASCIMENTO BARROS, Sandy Nara; SALES, Tâmara Regina Reis; DE FÁTIMA PIOVESAN, Angélica. Transtornos do Neurodesenvolvimento e Neurociência Educacional: Uma Revisão Sistemática. Semana de Pesquisa da Universidade Tiradentes-SEMPESq, 2018, 18.

FONSECA, Vitor da. Importância das emoções na aprendizagem: uma abordagem neuropsicopedagógica. Revista Psicopedagogia, v. 33, n. 102, p. 365-384, 2016. GADIA, Carlos A.; TUCHMAN, Roberto; ROTTA, Newra T. Autismo e doenças invasivas de desenvolvimento. Jornal de pediatria, 2004, 80.2: 83-94.

GIARDINETTO, A. Educação do aluno com autismo: um estudo circunstanciado daexperiência escolar inclusiva e as contribuições do currículo funcional natural. 2009. Tese (Doutorado em Educação) - Programa de Pós-Graduação em Educação da Faculdade de Filosofia e Ciências da Universidade Estadual Paulista, Marília, 2009. Disponível em:

<http://www.athena.biblioteca.unesp.br/exlibris/bd/bma/33004110040P5/2009/giardinet to_arsb_dr_mar.pdf>. Acesso em: 19 abril 2019.

GIL, A. C. Como classificar as pesquisas? In. Como elaborar projetos de pesquisa. - 4. Ed.-São Paulo: Atlas, 2002. p.42 GOLDBERG, Karla. A percepção do professor acerca do seu trabalho com crianças portadoras de autismo e síndrome de Down: um estudo comparativo. 2002.

KLIN, Ami. Autismo e síndrome de Asperger: uma visão geral Autism and Asperger syndrome: an overview. Rev Bras Psiquiatr, v. 28, n. Supl I, p. S3-11, 2006.

Kupfer, M.C.M (2001). Educação para o futuro. Psicanálise e educação. São Paulo: Escuta. $2^{\text {a }}$ Ed. 
MAIOR, Izabel. História, conceito e tipos de deficiência. Portal do Governo do Estado de São Paulo. Disponível em: http://violenciaedeficiencia. sedpcd. sp. gov. br/pdf/textosApoio/Texto1. pdf. Acesso em, 2018, 12.

MARTINOTO, LisianeBarcarolo; KORTMANN, Gilca Maria Lucena. O ENSINO ESTRUTURADO COMO EDUCAÇÃO E PROMOÇÃO EM SAÚDE PARA CRIANÇAS COM TRANSTORNO DO ESPECTRO AUTISTA-TEA. SEFIC 2015, 2017.

MARTINS, Mara Rubia Rodrigues et al. Inclusão de alunos autistas no ensino regular: concepções e práticas pedagógicas de professores regentes. 2007.

MEIMES, Maíra Ainhoren; SALDANHA, Helena Castro; BOSA, Cleonice Alves. Adaptação materna ao transtorno do espectro autismo: relações entre crenças, sentimentos e fatores psicossociais. Psico, v. 46, n. 4, p. 412-422, 2015.

OLIVEIRA, Rafaela Machado. O papel do Profissional de Apoio Escolar na inclusão da criança autista na educação infantil. Rafaela Machado Oliveira. Brasília: UnB. 2018. p.144.

PARRA, L. S. Atando laços e desatando nós: reflexões sobre a função do acompanhamento terapêutico na inclusão escolar de crianças autistas. 2009. $153 \mathrm{f}$. Dissertação (Mestrado em Psicologia Clínica e Cultura) - Universidade de Brasília, Brasília, 2009.

PORCINO, José Marciel Araújo; Oliveira, Tessya Hyanna Almeida; Caiana, Rayane Alexandrino; Pereira, Valéria, Amanda Jerônimo; Diniz, George Carneiro; Porcino, Jadcely Maria Viturino Serafim; Andrade, Joedna Vasconcelos Menezes. Aspectos neuropsicológicos de criança com transtorno do espetro do autismo. Revista Multidisciplinar do Nordeste Mineiro. Edição 2018-2(p.243-262) DOI 10.17648/2178$\underline{6925}$

PRESTES, M. A. Diagnóstico de autismo na rede pública de ensino do DF: um estudo exploratório da situação atual. Dissertação (Mestrado em Educação) Universidade Católica de Brasília, 2009.

RAMIRES, Vera Regina Röhnelt, et al. Saúde mental de crianças no Brasil: uma revisão de literatura. Interação em Psicologia, 2009, 13.2.

RIBAS, Dorotéa Loes; TERRA, Marlene Gomes; ERDMANN, Alacoque Lorenzini. 0 PARADIGMA COMPLEXO: Um Novo Olhar Para a Saúde Mental. Revista Contexto \& Educação, 2005, 20.73-74: 131-142.

SANCHES, Isabel Rodrigues; SIQUEIRA, Leni Porto Costa. A inclusão escolar e o transtorno do espectro do autismo. Comunicações, 2016, 23.3: 167-183.

SERRA, D. (2010). Sobre a inclusão de alunos com autismo na escola regular. Quando o campo é quem escolhe a teoria. Revista de Psicologia, 1(2), 163-176.

SALOMAO, P. E. A. et al. As Tecnologias de Informação e Comunicação (TIC) no ensino superior. Revista Multidisciplinar do Nordeste Mineiro, v. 1, 2018.

TAMANAHA, Ana Carina; PERISSINOTO, Jacy; CHIARI, Brasilia Maria. Uma breve revisão histórica sobre a construção dos conceitos do autismo infantil e da síndrome de Asperger. Revista da Sociedade Brasileira de Fonoaudiologia, 2008. 
Revista Multidisciplinar do Nordeste Mineiro, v1. 2020/01

ISSN 2178-6925

Human Services. Retirado em 21 de abril de 2019, de http://www.surgeongeneral.gov/library/mentalhealth/toc.html\#chapter3.

VIEIRA, A. M., et al. Saúde mental na escola. Estanislau GM, Bressan RA, org. Saúde mental na escola: o que os educadores devem saber. Porto Alegre: Artmed, 2014, 13-24.

Revista Multidisciplinar do Nordeste Mineiro, v...., n..., p. 01-X, 2018/02 ISSN 2178-6925 / doi.org/10.17648/2178-6925 